\title{
Cranial Transposition and Revascularization of Autologous Omentum: A Novel Surgical Technique for Bypassing the Blood Brain Barrier after Resection of Recurrent Glioblastoma Multiforme
}

\section{Omer Doron}

Lenox Hill Hospital https://orcid.org/0000-0002-2978-6639

Tom Chen ( $\nabla$ dr.tomchen@icloud.com )

Lenox Hill Hospital

Tamika Wong

Lenox Hill Hospital

Amy Tucker

Lenox Hill Hospital

\section{Peter Constantino}

Lenox Hill Hospital

\section{Robert Andrews}

Lenox Hill Hospital

David J Langer

Lenox Hill Hospital

John A Boockvar

Lenox Hill Hospital

\section{Research Article}

Keywords: Blood Brain Barrier, High Grade Glioma, Recurrent glioblastoma multiforme, Omental cranial free Flap Transposition, temporoparietal Fascial Flap, Revascularization, Milky spots

Posted Date: December 22nd, 2021

DOI: https://doi.org/10.21203/rs.3.rs-1160435/v1

License: (c) (i) This work is licensed under a Creative Commons Attribution 4.0 International License. Read Full License 


\section{Abstract}

Background: Glioblastoma multiforme (GBM) patients continue to suffer a poor prognosis. The blood brain barrier (BBB) comprises one of the obstacles for therapy, creating a barrier that decreases the bioavailability of chemotherapeutic agents in the central nervous system. Previously, a vascularized temporoparietal fascial scalp flap (TPFF) lining the resection cavity was introduced in a trial conducted in our institution, in newly-diagnosed GBM patients in an attempt to bypass the BBB after initial resection. In this paper, we report on a new technique to bypass the BBB after re-resection and potentially to allow tumor antigens to be surveilled by the immune system .

Objective: Assess the feasibility of performing a cranial transposition and revascularization of autologous omentum after re-resection of GBM.

Methods: Laparoscopically harvested omental free flap was transposed to the resection cavity by a team consisting of neurosurgeons, otolaryngologists, and general surgeons. This was done as part of a single center, single arm, open-label, phase I study.

Results: Autologous abdominal omental tissue was harvested laparoscopically on its vascularized pedicle in 2 patients, transposed as a free flap, revascularized using external carotid artery, and carefully laid into the tumor resection cavity. Patients did well postoperatively returning to baseline activities. Graft viability was confirmed by cerebral angiogram.

Conclusion: Omental cranial transposition of a laparoscopically harvested, vascularized flap, into the cavity of re-resected GBM patients is feasible and safe in the short term. Further studies are needed to ascertain whether such technique can improve progression free survival and overall survival in these patients.

\section{Introduction}

Glioblastoma multiforme (GBM) accounts for over $50 \%$ of primary malignant brain tumors in adults, and it is the most malignant form of high grade gliomas [(Taylor, Brzozowski, and Skelding 2019)]. Currently, median survival is poor, at 12-15 months, and progression free survival is approximately 5 to 6 months. The lack of chemotherapeutic agents which can penetrate beyond the blood brain barrier, remains a major research target aimed at advancing the care of GBM patients and potentially affecting survival [(Wang et al. 2019)].

Recently, our group opened a trial in which a vascularized temporoparietal fascial scalp flap (TPFF) was used to line the resection cavity, in an attempt to bypass the BBB[(Patel et al. 2020)]. By applying a harvested local scalp flap, we hypothesized that new vessels arising from the flap tissue may create a barrierless vascular bed that's in close contact with the microenvironment of tumor resection cavity thus allowing greater intracranial bioavailability of systemically administered chemotherapeutic agents. [(Chang et al. 2017)] 
In the current study (termed "Laparoscopically Harvested Omental Free Tissue Autograft to Bypass the Blood Brain Barrier in Human Recurrent Glioblastoma Multiforme", Clinical Trials Identifier: NCT04222309), we have targeted patients suffering from recurrent GBM. In these patients, we hypothesized that direct revascularization of an autologous omental free flap placed intracranially in contact with the tumor microenvironment may enable BBB bypass while also allowing lymph nodes in the omentum (so called "milky spots) to have access to tumor associated antigens. We hypothesized that the additional tissue qualities of omentum, including its highly vascularized nature, and pliability to fill varying sized defects may provide an accessible mean to circumvent the BBB. The exposure of the immune system to these antigens may allow better immune surveillance in the otherwise immunoprivileged central nervous system [(Chandra et al. 2011; Ezan et al. 2012; Meza-Perez and Randall 2017)].

In this paper, we report for the first time in human history, two cases in which autologous abdominal omental tissue, harvested laparoscopically as a re-vascularized pedicle, was transposed and revascularized and placed into the resection cavity of patients with recurrent GBM.

\section{Case Descriptions}

Cases described here were done as part of our single center, single arm, open-label, phase I study to assess the safety of laparoscopically harvested omental free flap transposed into the resection cavity of recurrent glioblastoma multiforme patients. Our trial was approved by the institutional review board (IRB). Inclusion criteria included recurrent GBM patients with a Karnofsky performance status (KPS) of 70 and who were deemed eligible to achieve a greater than $80 \%$ resection of enhancing disease. Informed consent was obtained and the surgery was performed by a team consisting of surgeons from neurosurgery, head and neck, and general surgery. consent to publish has been received from all participants.

Patient A was a 56-year-old female, diagnosed with IDH-wild type GBM 1.5 years ago who initially presented with a right temporoparietal mass, which was resected and treated with radiation and Temozolomide (TMZ). A year later, after a breakthrough seizure and radiographic evidence of further recurrence (Figure 1), the tumor was re-resected and patient was treated on a clinical trial with a single dose of intra-arterial bevacizumab with blood brain barrier disruption. However, because of a rapid recurrence, the patient was offered further resection with omental cranial free flap transposition.

Patient B, a 51-year-old physician, was diagnosed with IDH-wild type GBM 3 years ago who initially presented with a left frontal mass. He underwent two previous craniotomies at an outside institution. Despite adjuvant treatment consisting of radiation and TMZ, imaging unfortunately showed further disease progression (Figure 1).

\section{Surgical Procedure}


Under general anesthesia (TIVA protocol) and neuromonitoring, the patient was positioned in a supine position using a three-pin head holder, affixed to the operating room table (Figure 2).

For both patients a standard micro-neurosurgical operating techniques were utilized. 5 Aminolevulinic Acid (5 ALA) was used to maximize the extent of resection. Intra-operative neurophysiological monitoring was used to minimize the risk of neurological injury. The previous incision were slightly modified and extended in a linear fashion, while preserving the superficial temporal arteries. Access was obtained via a right temporoparietal craniotomy for patient $A$ while a left frontal craniotomy was utilized for patient $B$. After elevation of previous bone plate, total gross re-resection of tumor was carried out with aid of frameless navigation and 5 ALA dye. Temporary closure was achieved by stapling the scalp close during omental harvest.

The general surgery team then proceeded with the abdominal portion of the operation using Hasson supraumbilical entry with three additional trocars placed in the left and right abdomen. The abdomen was insufflated to a pressure of $15 \mathrm{mmHg}$ providing laparoscopic access to the abdominal cavity. Every effort was made to minimize the amount of time patient was placed in the Trendelenburg position, which was done for no more than 5 minutes at a time to avoid any increases in intracranial pressure. The omentum was positioned over the stomach exposing its natural attachments to the transverse colon. These natural attachments were carefully taken down with mostly sharp dissection and minimal amount of energy to avoid distorting the mesenchymal cells and vascularity of the omentum itself. The patient was then placed in reverse-Trendelenburg position and the natural attachments to the duodenum were taken down with blunt and sharp dissection. The gastroepiploic vessels supporting the omentum were divided from the stomach using successive firings of the Harmonic scalpel being careful not to encroach on the gastroepiploic pedicle itself or the stomach. Short gastric vessels were controlled with titanium clips as the pedicle was divided distally off the greater curvature of the stomach. Remaining attachments to the splenic flexure and duodenum were mobilized essentially freeing up the entire greater omentum except at the origin of the right gastroepiploic pedicle. Endoloop was used for proximal control and the pedicle was subsequently excised. The midline incision was slightly extended and the omentum was retrieved and passed off for back table preparation by the head and neck surgery team (Figure 2).

The head and neck surgery team proceeded with neck dissection as the right superior thyroid artery and a branch of the facial vein were dissected and prepared for microvascular anastomosis. Venous anastomosis was created using a $4 \mathrm{~mm}$ coupling ring while the arterial anastomosis was created using 80 nylon in interrupted fashion. Intravenous Indocyonine green (ICG) was administered by the anesthesiologist and an ICG laparoscopic camera was used to confirm blood flow to the flap. ICG was once again used to guide trimming of the flap which was subsequently performed to allowed it to fit into the resection cavity. This was performed with appropriate vascular type loads of the laparoscopic endoGIA stapling devices.

The omental flap was laid into the resection cavity with the gastroepiploic pedicle running down the neck just anterior to the tragus (Figure 3). ICG was used again to confirm blood flow to the omentum. Onlay dura-substitute (Duragen) was used to prevent postoperative CSF leak and the bone plate was 
subsequently reattached with a passageway for the omental pedicle to pass through. Particular care was taken to not overpack the resection cavity. After the omental cranial transposition was completed, closure was completed by replacing the native bone or by performing a cranioplasty using a titanium mesh, followed by closure of the neck and scalp areas using standard techniques. Doppler ultrasound was done every four hours for 4 days to ensure that the anastomosis remained patent post operatively.

\section{Post Operative Course}

Patient A was extubated on postoperative day one and was found to be neurologically intact with good dopplerable signals anterior to the tragus confirming omental vessel patency. Patient had return of bowel function on postoperative day 2 and her diet was advanced to regular. Postoperative MRI (Figure 4) showed no evidence of mass effect from omental flap. The remainder of her post-operative course was uncomplicated with stable neurological check-ups and the patient was subsequently discharged home on post-operative day 6 .

Patient B was extubated immediately after the procedure, but appeared slow to wake up with some dysphasia. CT head performed on the day of surgery showed mild omental flap-related mass effect, conservative management was done and mannitol was given to decrease intracranial pressure. A repeat CT head on postoperative day two showed mild hydrocephalus and a decision was made to place an external ventricular drain (EVD). Patient was treated over the next few days with a combination therapy consisting of steroids, CSF drainage, and mannitol. Patient was gradually weaned off the EVD by postoperative day 7 after returning to baseline neurological status. MRI head was subsequently performed (Figure 4) showing the pedicled flap filling up the tumor resection cavity with minimal midline shift.

At two month follow-up, both patients were functioning at baseline neurologically and resumed their preoperative level of activity of daily living. Patient A was initially started on oral lomustine but suffered significant bone marrow suppression thus she was subsequently switched to intravenous Bevacizumb (BV). Patient B resumed TMZ chemotherapy based on his local neuro-oncologists. For patient A, prior to starting IV bevacizumab we were able to cannulate the gastroepiploic vessels in the omental graft prior to delivering a dose of intra-arterial bevacizumab. This angiogram was done at approximately two months postop and it showed excellent graft patency (Figure 5). Interval MRI head showed stable residual enhancing disease. She is currently receiving biweekly IV Bevacizumab with follow up imaging every two months.

\section{Discussion}

Despite numerous efforts in recent decades, GBM continues to portend a poor prognosis with median survival of only 12-15 months for IDH wild type patients. Recurrences occur locally in $90 \%$ of cases and 
on average a few months after initial multimodal treatment [(Kirkpatrick et al. 2017; Mallick et al. 2016)]. Therefore new strategies are desperately needed in pursuit of a better outcome.

The blood brain barrier (BBB) comprises one of the primary problems for GBM therapy. As such, multiple attempts have been made to bypass it both in vitro and in vivo [(Kang and Adamson 2015; Shergalis et al. 2018)].

Lately, as part of a trial conducted in our department, we have published the technical feasibility of using a rotated, vascularized temporoparietal flap (TPF) combined with primary resection in newly-diagnosed GBM patients [3]. As the majority of GBM re-occurs within two centimeters of the primary resection cavity, the technique described here is presented as an alternative pathway to bypass the BBB using a wellestablished omental free flap [(Costantino et al. 2017; Eleazar et al. 2014; Mazzaferro et al. 2018)].

At our institution, laparoscopically harvested omental free flaps are commonly used to fill surgical cavities after resection and radiation of head and neck cancers such as squamous cell carcinoma(Costantino et al. 2017). Omentum with its rich vascularity can help wound healing in cases of osteoradionecrosis of the head and neck [(K. et al. 2013; Mclntyre et al. 2017; Shen and Shen 2003)].

Goldsmith and his team have shown that transposed omentum onto the cortex of brain can ignite vessel penetration into the cortex within 12 hours. In 1990, the feasibility of omental vessels to form collaterals with the subarachnoid vessels was shown in pre-clinical GBM models [(Berger et al. 1990)]. We hypothesized that these new vascular structures would lack an intact BBB. We hope that the permeability of the blood vessels will allow for improved delivery of post-operative chemotherapeutics agents. Additionally, by placing omental autologous lymph nodes (milky spots) with immune cells (macrophages, dendritic, B and T cells) into the surgical cavity, perhaps tumor associated antigens will have a better chance at being recognized by the hosts immune system[(Liu et al. 2021; Mebius 2009)]. We recognize that the potential risks of this procedure include the added length of the surgical resection, the risk of seizures from the presence of a graft in the resection cavity, and the distinct possibility that tumor could hijack the graft vasculature and grow even more aggressively. Although our trial is at its infancy, we have yet to see the aforementioned complications in our TPF patients.

This report is the first of its kind to describe the feasibility of this technique in human. We aim to prove that this surgical technique is safe for patients with recurrent GBM and we hope it can improve survival outcomes for these patients with devastating recurrences.

\section{Conclusions}

Omental cranial transposition of a laparoscopically harvested, vascularized flap, into the resection cavity of re-resected GBM patients is feasible and safe in the short term. Further studies are needed to ascertain whether such technique can improve progression free survival and overall survival in patients with recurrent GBM. 


\section{Declarations}

Funding : this study has was not supported by any grant or otherwise funded, by any party.

Conflicts of interest/Competing interests: None.

\section{References}

1. Berger MS et al (1990) "Omental Transposition to Bypass the Blood Brain Barrier for Delivery of Chemotherapeutic Agents to Malignant Brain Tumours: Preclinical Investigation." In The Omentum

2. Chandra A et al (2011) "The Anti-Inflammatory and Antibacterial Basis of Human Omental Defense: Selective Expression of Cytokines and Antimicrobial Peptides." PLOS ONE

3. Chang PD et al (2017) "Predicting Glioblastoma Recurrence by Early Changes in the Apparent Diffusion Coefficient Value and Signal Intensity on FLAIR Images." American Journal of Roentgenology

4. Costantino PD et al (2017) "The Laparoscopically Harvested Omental Free Flap: A Compelling Option for Craniofacial and Cranial Base Reconstruction." Journal of Neurological Surgery, Part B: Skull Base

5. Eleazar TF et al (2014) "Greater Omentum Free Flap for Reconstruction of Extensive Lesions of the Scalp." Revista Venezolana de Oncología

6. Ezan P et al (2012) "Deletion of Astroglial Connexins Weakens the Blood-Brain Barrier." Journal of Cerebral Blood Flow and Metabolism

7. Fujino K et al (2013) "Single Port Laparoscopic Harvest of Omental Flap for Reconstruction of Refractory Wound." Surgical Endoscopy and Other Interventional Techniques

8. Kang JH (2015) and Cory Adamson. "Novel Chemotherapeutics and Other Therapies for Treating High-Grade Glioma." Expert Opinion on Investigational Drugs

9. Kirkpatrick JP, Nadia N, Laack HA, Shih (2017) and Vinai Gondi. "Management of GBM: A Problem of Local Recurrence." Journal of Neuro-Oncology

10. Liu M, Silva-Sanchez A, Randall TD, and Selene Meza-Perez (2021) “Specialized Immune Responses in the Peritoneal Cavity and Omentum." Journal of Leukocyte Biology

11. Mallick S, Benson R, Hakim A, Rath GK (2016) "Management of Glioblastoma after Recurrence: A Changing Paradigm." Journal of the Egyptian National Cancer Institute

12. Mazzaferro D et al (2018) "The Omental Free Flap-A Review of Usage and Physiology." Journal of Reconstructive Microsurgery

13. McIntyre BC, Lobb D, Navarro F, and James Nottingham (2017) “Laparoscopic Free Omental Flap for Craniofacial Reconstruction: A Video Article Demonstrating Operative Technique and Surgical Applications." Journal of Craniofacial Surgery28(2)

14. Mebius RE (2009) "Lymphoid Organs for Peritoneal Cavity Immune Response: Milky Spots." Immunity 
15. Meza-Perez, Selene, Randall TD (2017) "Immunological Functions of the Omentum." Trends in Immunology

16. Patel NV et al (2020) "Vascularized Temporoparietal Fascial Flap: A Novel Surgical Technique to Bypass the Blood-Brain Barrier in Glioblastoma." World Neurosurgery

17. Shen YM (2003) and Zu Yao Shen. "Greater Omentum in Reconstruction of Refractory Wounds." Chinese Journal of Traumatology - English Edition

18. Shergalis A et al (2018) "Current Challenges and Opportunities in Treating Glioblastomas." Pharmacological Reviews

19. Taylor OG, Joshua S, Brzozowski, Skelding KA (2019) “Glioblastoma Multiforme: An Overview of Emerging Therapeutic Targets." Frontiers in Oncology

20. Wang D, Wang C, Wang L, Chen Y (2019) "A Comprehensive Review in Improving Delivery of SmallMolecule Chemotherapeutic Agents Overcoming the Blood-Brain/Brain Tumor Barriers for Glioblastoma Treatment." Drug Delivery

\section{Figures}
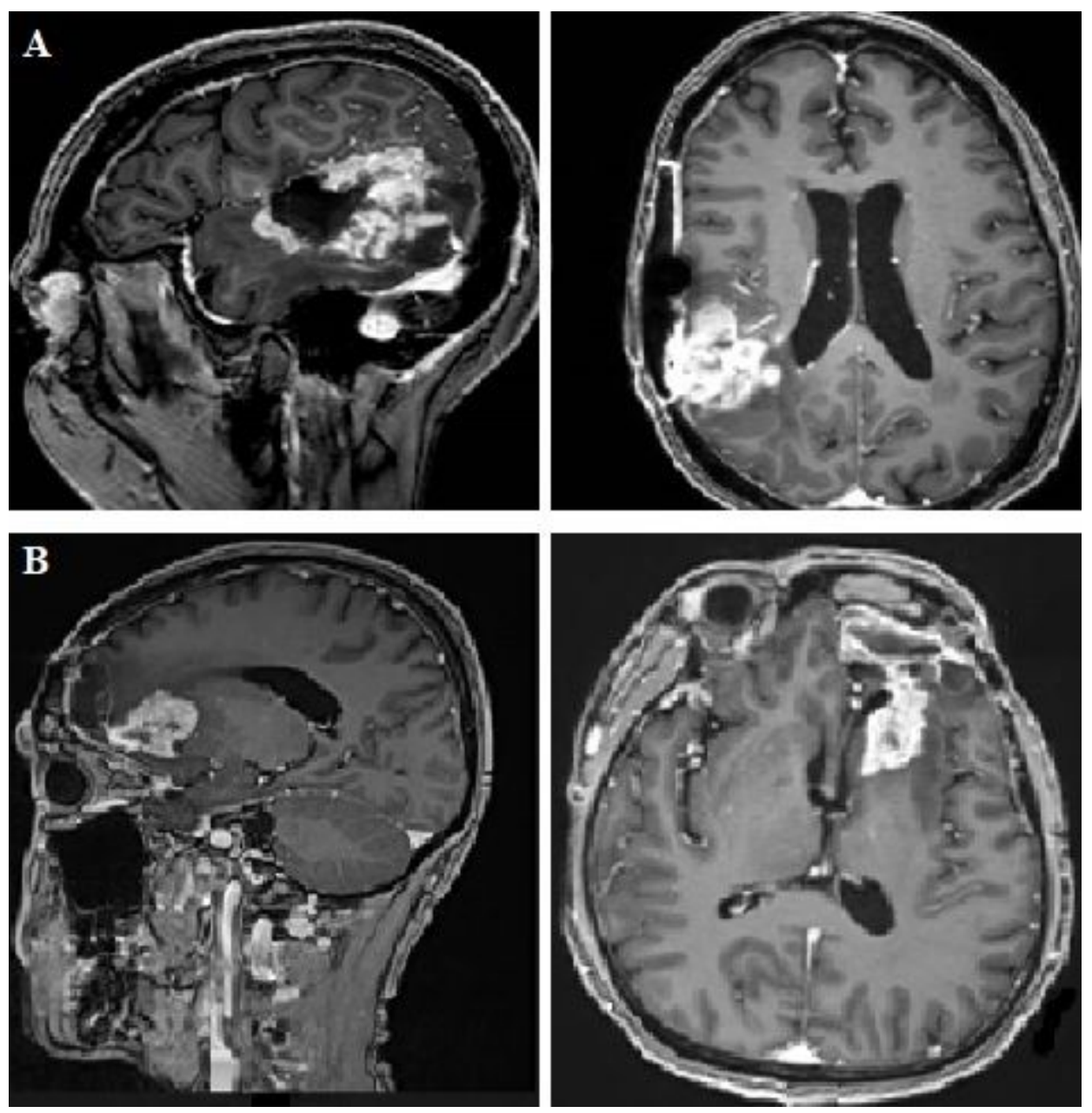

Figure 1 
Pre-Operative MRI T1 with gadolinium showing tumor recurrence. A (top), case 1 . B (bottom), case 2. (B).
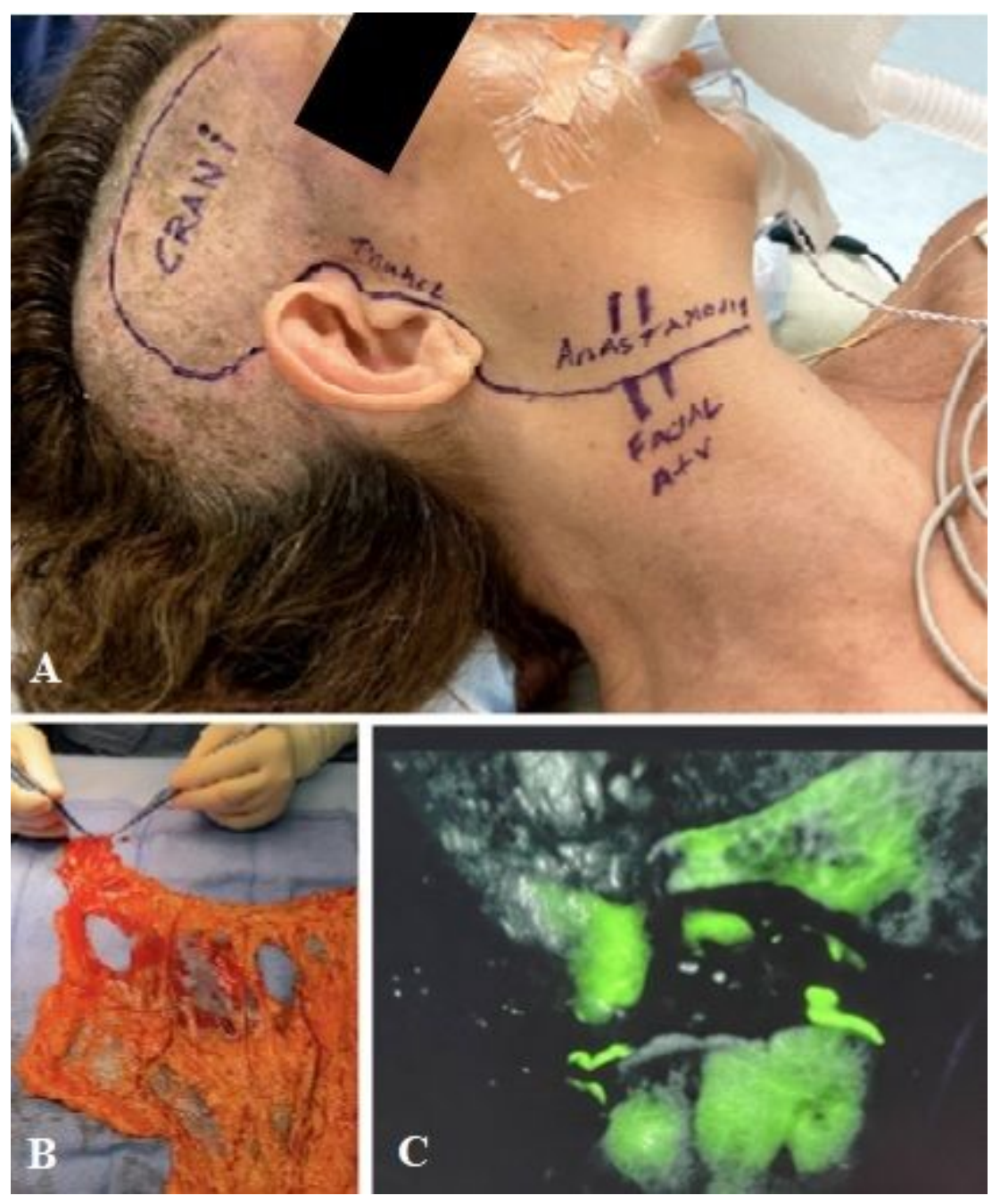

Figure 2

A, Skin-marking incision including the neck and access to the cranium. B, Omentum harvested as a pedicle with natural blood vessels . C, ICG within the omentum after anastomosis completed to neck vessels confirming vascularized pedicle 

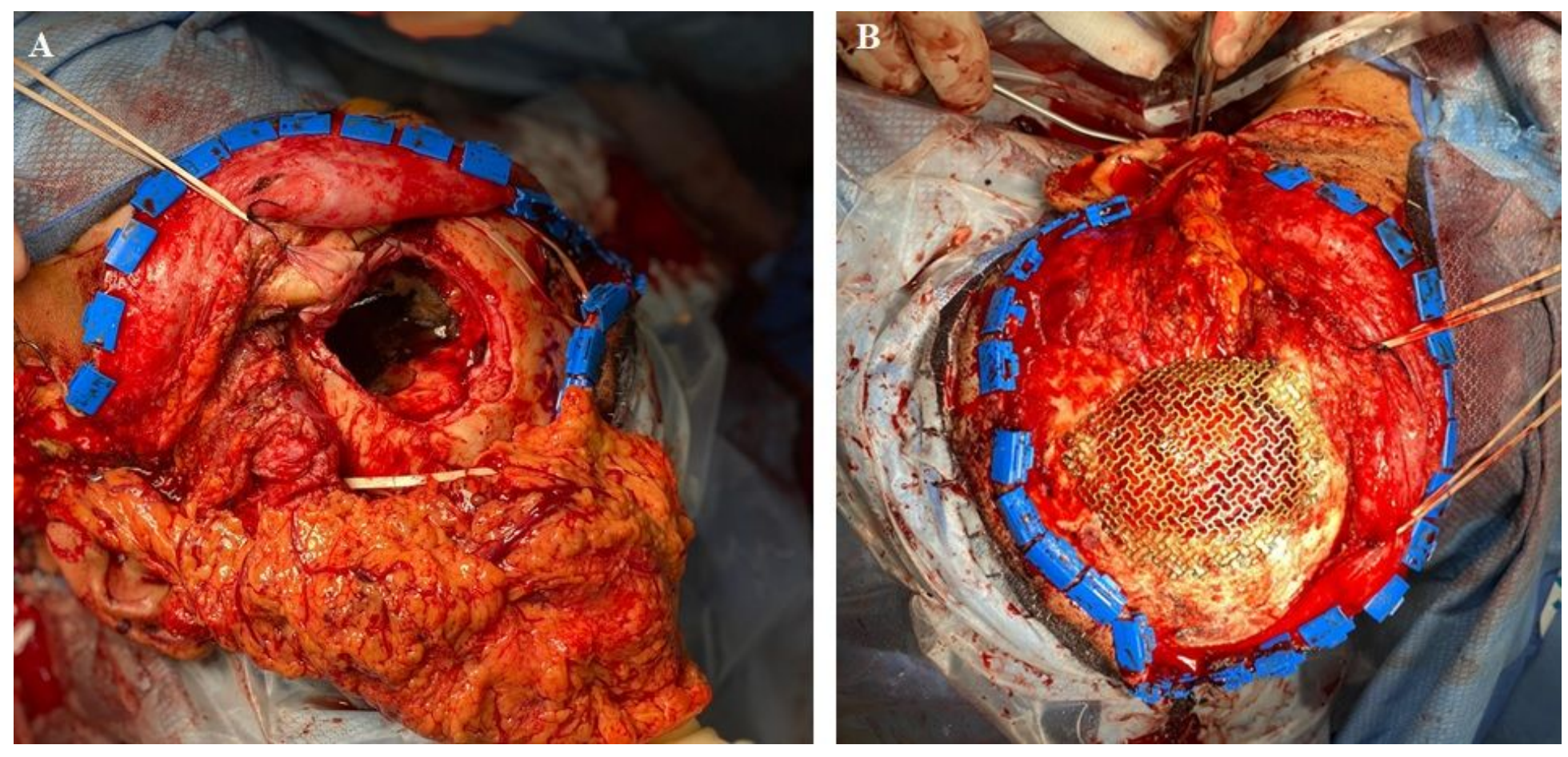

\section{Figure 3}

A, Patient's omental flap intra-operatively placed in the resection cavity with a vascularized transposed pedicle extending from the neck cranially. B, Titanium mesh and closure of transposed flap. 


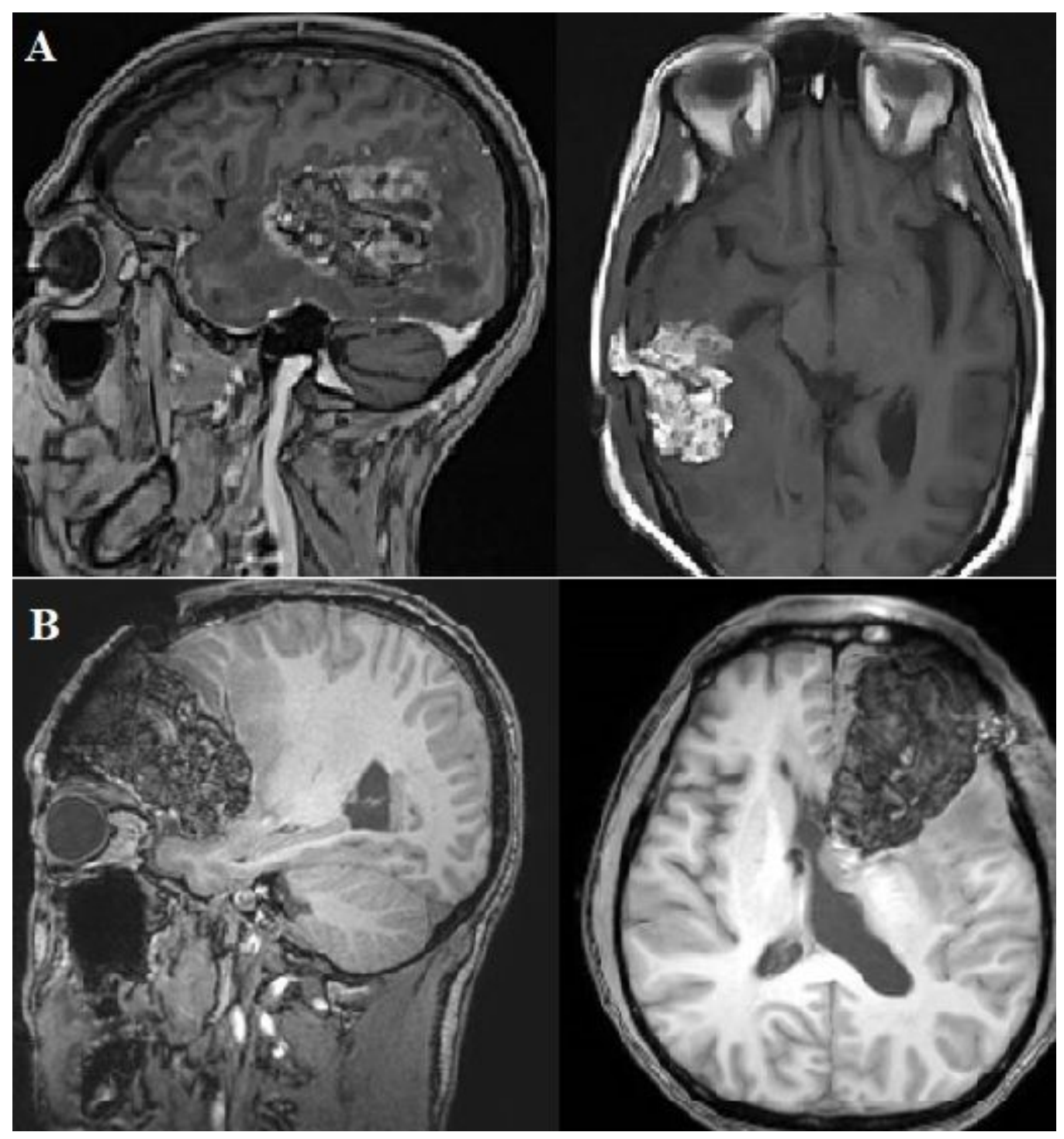

\section{Figure 4}

Post operative MRI scans, showing the flap with its vascular supply in the resection cavity. Top, Case 1 (A). Bottom, Case 2 (B). 

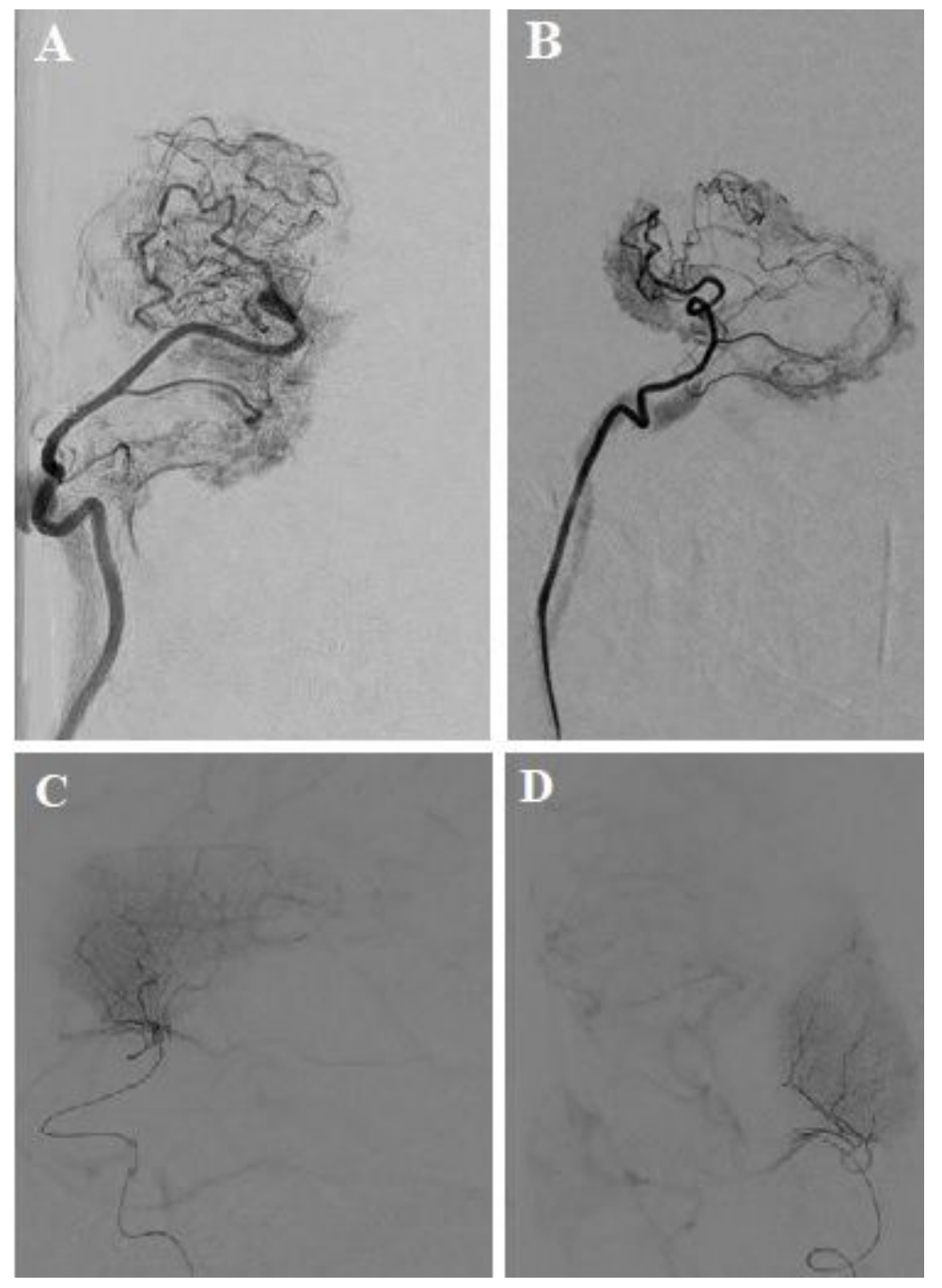

\section{Figure 5}

Angiographic view of the graft within tumor cavity in case 1 , showing tumor and omental flap blush. A, left external carotid injection in an anterior-posterior view. B, left external carotid injection in a lateral view. C, Left middle cerebral artery M1 segment injection, lateral view. D, Left middle cerebral artery M1 segment injection, anterior-posterior view.

\section{Supplementary Files}

This is a list of supplementary files associated with this preprint. Click to download.

- Editor.mp4 